\title{
Long-term effects of short hairpin RNA-targeted human telomerase reverse transcriptase on suppression of SGC-7901 cell proliferation by inhibition of telomerase activity
}

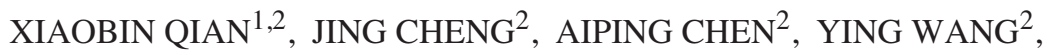 \\ YAN TAO ${ }^{2}$, JIANPING CAO ${ }^{3}$ and ZHENQING FENG ${ }^{1}$ \\ ${ }^{1}$ Department of Pathology, Nanjing Medical University; ${ }^{2}$ Department of Pathology, \\ School of Medicine, Jiangsu University; ${ }^{3}$ National Institute of Parasitic Disease, \\ Chinese Center for Disease Control and Prevention, Shanghai, P.R. China
}

Received August 9, 2007; Accepted November 9, 2007

\begin{abstract}
Human telomerase reverse transcriptase (hTERT) is an attractive target for cancer gene therapy. However, the poor inhibition of telomerase and a time lag between the inhibition and arrest of cell proliferation limits its use in cancer gene therapy. RNA interference (RNAi) has been proposed as a potential technique for the treatment of cancer with a long-term gene silencing response induced by short hairpin RNA (shRNA). To investigate the long-term effects of telomerase inhibition through the down-regulation of the hTERT gene and the potential role of hTERT in cancer gene therapy, we constructed an shRNA-directed hTERT-expressing vector and introduced it into SGC-7901 cells. A population of cells that stably expressed the shRNA was selected by G418 and continuously cultured in a medium with half the antibiotic concentration for 3 months and the anti-proliferation effects of shRNA-targeted hTERT were then detected. The results showed that shRNA-targeted hTERT significantly inhibited cell proliferation and increased cell apoptosis by down-regulating hTERT expression, thus decreasing telomerase activity. These findings suggest that shRNAtargeted $h T E R T$ has long-term anti-proliferation effects on SGC-7901 cells, and it is a potential approach in telomerasebased gene therapy.
\end{abstract}

\section{Introduction}

RNA interference (RNAi) technology has rapidly developed in recent years. The application of RNAi to mammalian cells

Correspondence to: Dr Zhenqing Feng, No. 140 Hanzhong Road, Nanjing City, Jiangsu Province 210029, P.R. China

E-mail: fengzhenqing@njmu.edu.cn

Key words: RNA interference, human telomerase reverse transcriptase, cancer gene therapy has revolutionized the field of functional genomics and this application has the therapeutic potential to down-regulate the expression of related disease-causing genes. Some strategies of RNAi have been approved in gene therapy for leukemia (1), AIDS (2) and viral hepatitis $(3,4)$.

Small interfering RNA (siRNA) is usually adopted for effective gene-specific RNA silencing. However, the silencing response induced by siRNA is transient, lasting just 3-5 days in culture and this restricts its application in gene therapy. Conversely, short hairpin RNA (shRNA) can generate a long-term gene-silencing response. The first step in the method using shRNA involves its expression from plasmid vectors. These vectors utilize RNA polymerase-III promoters H1 or U6 to express shRNA which is then processed into 21-bp siRNA by a Dicer enzyme. Subsequently, it results in mRNA degradation and silencing of the cognate gene expression.

Telomerase is a particularly attractive target for cancer therapy. Previous studies have shown that $\sim 90 \%$ of malignant tumor cells demonstrate telomerase activity, whereas most normal cells do not (5). Many studies have also demonstrated a strong correlation between telomerase activity and malignancy. Consequently, some researchers have indicated that human telomerase reverse transcriptase (hTERT) expression and telomerase activity play an important role in the immortality of tumor cells. Therefore, it is reasonable to consider that telomerase, particularly hTERT, serves as a good target for cancer therapy and the use of telomerase inhibitors may be a powerful approach in cancer therapy. Telomerase inhibitors can suppress the growth of cancer cells, and the adverse effects of this therapy on normal cells are limited as compared to routine chemotherapy. However, a challenge for the development of useful telomerase inhibitors is to overcome the long-time lag between telomerase inhibition and the arrest of cell proliferation.

Therefore, in this study, we constructed an shRNA-directed hTERT-expressing vector and introduced it in SGC-7901 cells in order to investigate the long-term effects of telomerase inhibition by regulation of the $h T E R T$ gene. We also evaluated its potential role in cancer gene therapy. 
Table I. The sequence targeting hTERT.

Psi-control
Sense strand: CACCTATAACAACGGTAG
Antisense strand: CTACCGTTGTTATAGGTG
Psi-hTERT1: (NM AB086950; nucleotides 2656-2674; GC content, 38.1\%)
Sense strand: 5'-TCATCAGCAAGTTTGGAAG-3'
Antisense strand: 5'-CTTCCAAACTTGCTGATGA-3'
Psi-hTERT2: (NM AB086950; nucleotides 2379-2397; GC content, 33.3\%)
Sense strand: 5'-TGGTGGATGATTTCTTGTT-3'
Antisense strand: 5'-AACAAGAAATCATCCACCA-3'

\section{Materials and methods}

Cell lines. The human gastric carcinoma cell line SGC-7901 was obtained from the Institute of Cell Biology, Chinese Academy of Sciences (Shanghai, China).

Reagents. The following reagents were obtained: Dulbecco's modified Eagle's medium (DMEM) from Gibco (Grand Island, NY); new bovine serum (NBS) from Minhai Bio-engineering C. (Lanzhou, China); a lined, empty expression siRNA vector (pSilencer 2.1-U6 neo siRNA expression vector) from Ambion Corporation (Ambion, USA); restriction enzymes and T4 DNA ligase from Takara Company and cell transfection reagent, SOFO liposome, from Sofast Company (Xiamen, China). The antibodies against hTERT and GAPDH were obtained from Boster Biological Technology (Wuhan, China) and horseradish peroxidase (HRP)-conjugated secondary antibody, from Jackson Immuno-Research Laboratories (West Grove, PA) and the electrochemiluminescence (ECL) reagents, from Amersham Biosciences (Buckinghamshire, UK). The oligonucleotides and PCR primers were chemically synthesized and DNA sequencing was performed by the Shanghai Invitrogen Company (China).

Cell culture. SGC-7901 cells were grown in DMEM containing $10 \% \mathrm{NBS}, 100 \mu \mathrm{g} / \mathrm{ml}$ penicillin and $100 \mu \mathrm{g} / \mathrm{ml}$ streptomycin at $37^{\circ} \mathrm{C}$ in a $5 \% \mathrm{CO}_{2}$ humidified incubator.

Construction of shRNA-expressing plasmid-targeting hTERT. According to the information on the Ambion website, at http://www.Ambion.com/techlib/misc/siRNA-design.html, we designed the short hairpin structure of the shRNA transcript template with a length of $63 \mathrm{bp}$, including BamHI and HindIII restriction endonuclease sites at each terminal. A 19-bp target sequence targeting different points of hTERT, a 9-bp loop sequence at the center of the inverse repetition target sequence and a $5 \mathrm{~T}$ as transcript terminator of RNA polymerase-III were also designed. Oligonucleotides were chemically synthesized by the Shanghai Invitrogen Company. Different pairs of shRNA template oligonucleotides were annealed and ligated into the pSilencer 2.1-U6 neo siRNA expression vector by using T4 DNA ligase. Then, we transformed DH5 $\alpha$ with the ligation products, picked the clones, isolated the plasmid DNA, digested the plasmid with
Table II. The PCR-primer sequence of hTERT and GAPDH.

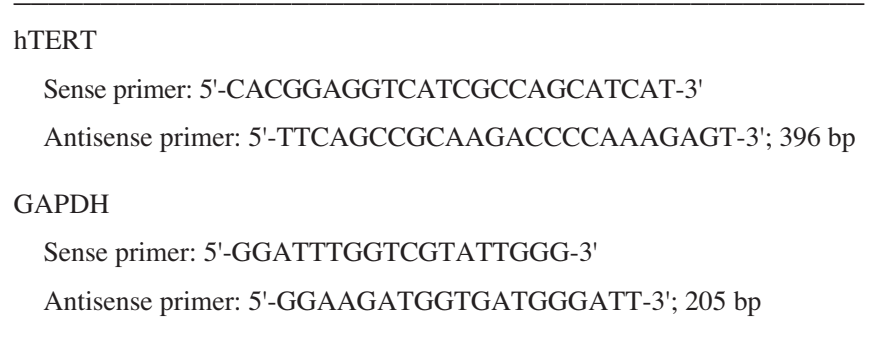

BamHI and HindIII to confirm the shRNA insert and sequenced the insert to confirm the existence of the required sequences. Three independent groups were designed for this experiment. One is the control group (psi-control), supplied by Ambion Corporation, which expresses shRNA with a limited homology to any known sequence in the human, mouse and rat genomes. The other two experimental groups were designated as psi-hTERT1 and 2 and had different 63 bp short fragments of oligonucleotides targeted at the corresponding sequences in hTERT. Their sequences are listed in Table I.

Transfection of the shRNA-targeted hTERT expression plasmid into SGC-7901 cells. SGC-7901 cells were plated in a 6 -well plate at a density estimated to reach $80 \%$ confluence after $24 \mathrm{~h}$. Transfection was performed using SOFO liposome according to the manufacturer's protocol. SOFO liposome $(6 \mu \mathrm{l})$ and $2 \mu \mathrm{g}$ shRNA-targeted hTERT-expressing plasmid were separately diluted with $50 \mu 1$ serum-free medium. The diluted SOFO liposome was added to the diluted plasmid and incubated at room temperature for $20 \mathrm{~min}$. The entire mixture was then added into the different wells resulting in varying plasmid concentrations in a total volume of $2.0 \mathrm{ml}$. To select the SGC-7901 cells that stably expressed shRNA, the cells were treated with $400 \mu \mathrm{g} / \mathrm{ml} \mathrm{G} 418$ for 14 days until all the non-transfected control cells were killed. The cells continued to be cultured with $200 \mu \mathrm{g} / \mathrm{ml} \mathrm{G} 418$ for 3 months and the medium was replaced every 3 days during the course of this culture. At the end of the culture period, the cells of the three different groups were collected to evaluate their hTERT expression levels, telomerase activity, cell growth rate and apoptosis.

The hTERT mRNA level was quantified by a real-time PCR method. Total RNA from the cells of the different groups was extracted using a Trizol reagent (Invitrogen, USA) and cDNA was synthesized in $20 \mu \mathrm{l}$ of reaction volume containing $4 \mu \mathrm{g}$ total RNA and by using SuperScript ${ }^{\mathrm{TM}}$ II RT (Invitrogen). The conditions of real-time PCR were as follows: $1 \mu \mathrm{l}$ of 1:1000 x SYBR-Green I, $0.5 \mu 1 \mathrm{cDNA}, 10 \mathrm{pmol}$ of each primer, $2 \mathrm{mM} \mathrm{MgCl}_{2}, 2.5 \mu 1200 \mathrm{nM}$ dNTP and 1 unit of Taq DNA polymerase were included in a $25 \mu 1$ volume of reaction mixture. To compensate for variations in the amount of RNA input and the efficiency of reverse transcription, an endogenous housekeeping gene (glyceraldehyde-3-phosphate dehydrogenase, GAPDH) was also quantified and used to normalize the results. The specific primers of hTERT and GAPDH are shown in Table II. The thermal cycle profile for PCR was $94^{\circ} \mathrm{C}$ for $2 \mathrm{~min}$ followed by 35 cycles of $30 \mathrm{sec}$ each at $94^{\circ} \mathrm{C}$, 
$45 \mathrm{sec}$ at annealing temperature $\left(59^{\circ} \mathrm{C}\right.$ for hTERT and $55^{\circ} \mathrm{C}$ for GAPDH), $45 \mathrm{sec}$ at $72^{\circ} \mathrm{C}$ and an additional $10 \mathrm{~min}$ of incubation at $72^{\circ} \mathrm{C}$ after completion of the last cycle for extension.

To analyze the real-time RT-PCR results, the starting quantity of a specific mRNA in an unknown sample was determined by preparing a standard curve using known dilutions of the standard plasmid DNA. The amplified fragments of the hTERT and GAPDH genes were cloned into pGEM-vectors (Promega, USA) and DNA sequencing was performed. The standard curves were generated using serial dilutions of the pGEM-hTERT and -GAPDH vectors and were based on the linear relationship between the $\mathrm{Ct}$ value (corresponding to the cycle number at which a significant increase in the fluorescence signal was first detected) and the logarithm of the starting quantity. The amplification plot and the corresponding dissociation curves were examined for each sample. The data were then viewed and analyzed by Rotor-gene real-time analysis software (Rotor-Gene 2000, CR, Australia). The copy numbers of hTERT and GAPDH were obtained according to each standard curve. The ratio between the copy numbers of hTERT and GAPDH represented the normalized hTERT for each sample, and it was comparable to that of the other sample (6). The following formula was used: NhTERT $=$ (hTERT mRNA copies of the sample/ GAPDH mRNA copies of the sample) $x$ 100. All the experiments were performed in triplicate and the standard deviation was obtained.

Western-blot analysis of the hTERT protein. The SGC-7901 cells of the different groups were harvested, washed with phosphate-buffered saline (PBS) and treated with trypsin. The nuclear protein extract which, was obtained with a nuclear fractionation kit from BioVision, was fractionated in $12 \%$ SDS-PAGE. Electrophoresis was conducted at a voltage of $8 \mathrm{~V}$ for stacking and $15 \mathrm{~V}$ for protein separation. The gel contents were electrotransferred to a polyvinyl difluoride (PVDF) membrane and the membranes were blocked in $3 \%$ $(\mathrm{w} / \mathrm{v})$ bovine serum albumin in TBS-T for $1 \mathrm{~h}$ at room temperature. Subsequently, the membranes were incubated with primary and secondary antibodies at $4^{\circ} \mathrm{C}$ overnight and at room temperature for $50 \mathrm{~min}$, respectively, with three washes after each incubation. ECL reagents were used to detect the positive bands on the membranes. Equal volumes of solution A and B were mixed and added to the protein side of the membranes and incubated for $1 \mathrm{~min}$ at room temperature. The exposure time of the first film was $15 \mathrm{sec}$ and that of the second film was adjusted according to the intensity of the signal on the first film. An endogenous housekeeping gene, GAPDH, was also quantified and used to normalize hTERT. The bands on the films were scanned by Typhoon 9400 phosphoimager (GE Healthcare) and digitized using ImageQuant software (GE Healthcare). The ratio of hTERT versus GAPDH expression was used as a measurement of the hTERT level.

Telomerase activity assay. The TRAP-ELISA method was used to determine the telomerase activity. All steps were performed according to the instruction manual of the TRAPeze Telomerase ELISA detection kit (Chemicon Corporation,
USA). Cells $\left(5 \times 10^{5}\right)$ from the three different groups were harvested and pelleted. After washing with PBS, the pellets were re-suspended in $200 \mu 1$ lysis buffer (10 Mm Tris- $\mathrm{HCl}$, $1 \mathrm{mM} \mathrm{MgCl} 2,1 \mathrm{mM}$ EGTA, $0.1 \mathrm{mM}$ ß-mercaptoethanol, $0.5 \%$ CHAPS and $10 \%$ glycerol) on ice for $30 \mathrm{~min}$. A $2-\mu 1$ aliquot of the supernatant was used as the TRAP template after centrifuging at $14,000 \mathrm{rpm}$ at $4^{\circ} \mathrm{C}$ for $20 \mathrm{~min}$. The reaction mixture was incubated at $25^{\circ} \mathrm{C}$ for $30 \mathrm{~min}$ and 35 ampli-cycles of PCR were then carried out $\left(94^{\circ} \mathrm{C}\right.$ for $30 \mathrm{sec}$ and $59^{\circ} \mathrm{C}$ for $60 \mathrm{sec}$ in each cycle). The PCR products were assayed according to the manufacturer's protocol. The absorbance of the samples was measured with a microtiter plate reader at $450 \mathrm{~nm}$ and this procedure was repeated 3 times.

Measurement of apoptosis. An Annexin-V/PI apoptosis detection kit I (BD Biosciences, USA) was used to measure apoptosis. Cells from the three groups were harvested at a concentration of $10^{5} / \mathrm{ml}$ and washed twice with ice-cold PBS. The supernatant was discarded after centrifugation and the pellet was then re-suspended in $200 \mu \mathrm{l}$ binding buffer. To this solution, $10 \mu 1$ Annexin-V-FITC and $10 \mu 1$ propidium iodide (PI) were added and the solution was placed in the dark for $15 \mathrm{~min}$ at room temperature. Cell apoptosis was analyzed immediately using a FACSCalibur cytometer (BectonDickinson, USA) and CellQuest version $3.1 \mathrm{f}$ software (Becton-Dickinson).

Cellular proliferation activity assay. The cells from the different groups were trypsinized and seeded in 96-well plates at a density of $1.2 \times 10^{4} / 100 \mu 1$. The cells were cultured for 1,2 , 3,4 or 5 days. Cell proliferation was detected by the $3-(4,5-$ dimethylthiazol-2-yl)-2,5-dimethyl tetrazolium bromide (MTT) colorimetric method (7). The units of absorption (UA) were measured by using a photometer (Bio-tek MQX200) at a wavelength of $570 \mathrm{~nm}$.

Statistical analysis. All data were expressed as the mean \pm standard deviation (SD). Statistical analysis was performed by SPSS 13.0 and significance was defined as $\mathrm{P}<0.05$.

\section{Results}

The verification of recombinant plasmids and screening of the transient effect of shRNA-targeted hTERT. The construction scheme of the shRNA expression vector is shown in Fig. 1. Psi-hTERT1 and 2 vectors were digested by HindIII and BamHI and the digestion products were identified by $3 \%$ agarose gel electrophoresis. The sequences of the two vectors were confirmed by sequencing and were compared with the designed fragments. Prior to the long-term experiment, the transient effects of shRNA-targeted hTERT were tested. The hTERT mRNA expression was detected after $1.0 \mu \mathrm{g} / \mathrm{ml}$ of the shRNA-targeted hTERT was transfected into SGC-7901 cells and cultured for $48 \mathrm{~h}$. The results showed that psi-hTERT1 and 2 inhibited the hTERT mRNA expression (data not shown).

The effects of shRNA-targeted hTERT on the hTERT gene expression. To determine the effect of shRNA-targeted hTERT on the hTERT gene expression, real-time PCR and Western 

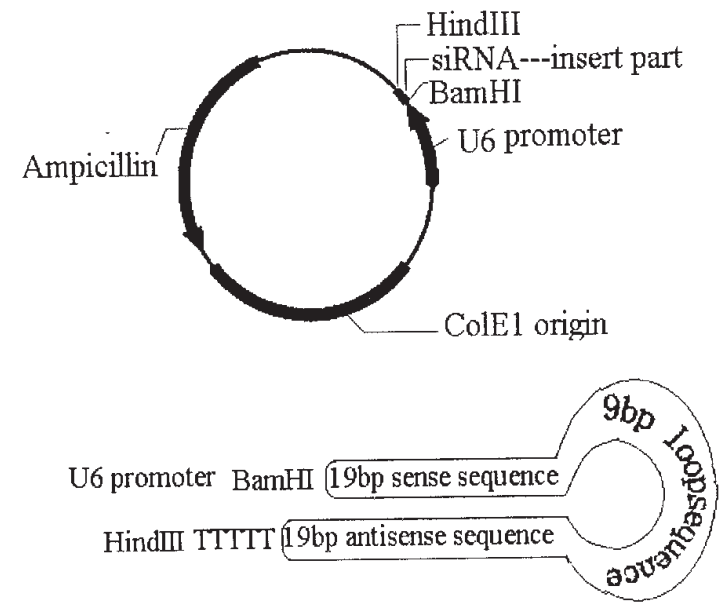

Figure 1. The construction scheme of an shRNA expression vector.

blot were used to examine the hTERT gene expression. After the SGC-7901 cells were treated with different shRNAtargeted hTERT at a dose of $1 \mu \mathrm{g} / \mathrm{ml}$ for 3 months, the results demonstrated that psi-hTERT1 and 2 inhibited the expression of hTERT at the mRNA and protein level, when compared with the psi-control. Furthermore, the two shRNA-targeted hTERT presented different abilities in inhibiting the $h T E R T$ expression whereby psi-hTERT1 showed a $75.1 \%$ reduction in the mRNA expression of hTERT and psi-hTERT2 showed an $85.9 \%$ reduction (Fig. 2A). The hTERT protein level also decreased in the psi-hTERT1 and 2 groups (Fig. 2B and C) when compared with the psi-control cells and there were significant differences $(\mathrm{P}<0.05)$.

The effects of shRNA-targeted hTERT on telomerase activity. Telomerase activity levels showed obvious changes after the SGC-7901 cells were treated with different shRNA-targeted hTERT at a dose of $1 \mu \mathrm{g} / \mathrm{ml}$ for 3 months. The psi-hTERT2 group showed a stronger inhibition of telomerase activity than psi-hTERT1. A $36.4 \%$ reduction in telomerase activity (from 2.09 to 1.33 ) in psi-hTERT1 and a 51.2\% reduction (from 2.09 to 1.02) in psi-hTERT2 (Fig. 3) was observed. There were significant differences in the telomerase activity among the psi-hTERT1 and 2, and psi-control groups $(\mathrm{P}<0.05)$.

The effects of the shRNA-targeted hTERT on the proliferation of SGC-7901 cells. After the SGC-7901 cells were treated with a different shRNA-targeted hTERT at a dose of $1 \mu \mathrm{g} / \mathrm{l}$ for 3 months, the growth of these cells was obviously inhibited by psi-hTERT1 and 2 (Fig. 4). After 2 days, compared with the psi-control cells, there were significant differences $(\mathrm{P}<0.05)$. A group of untreated cells was shown to estimate the effect of the psi-control group cells. There were no differences between the untreated group and psi-control.

shRNA-targeted hTERT induces apoptosis of SGC-7901 cells. The annexin-V/PI double staining method was used to measure cell apoptosis. After the SGC-7901 cells were treated with different shRNA-targeted hTERT at a dose of $1 \mu \mathrm{g} / \mathrm{ml}$ for 3 months, cell apoptosis increased in the cells treated with psi-hTERT1 and 2 but that of the viable cells decreased (Fig. 5).

\section{Discussion}

hTERT is an attractive target for cancer therapy. Human telomerase stabilizes telomere length, thereby contributing to unlimited cell proliferation (8-11) and playing an important role in cell proliferation and carcinogenesis. Research has shown that $70-95 \%$ of malignant tumors and a majority of cancer cell lines, but not most normal somatic cells, express relatively high levels of telomerase activity (5). Several in vitro studies have demonstrated that telomerase activity is inhibited in telomerase-positive cells and that such inhibition is associated with a reduction in cell viability, and the cell
A

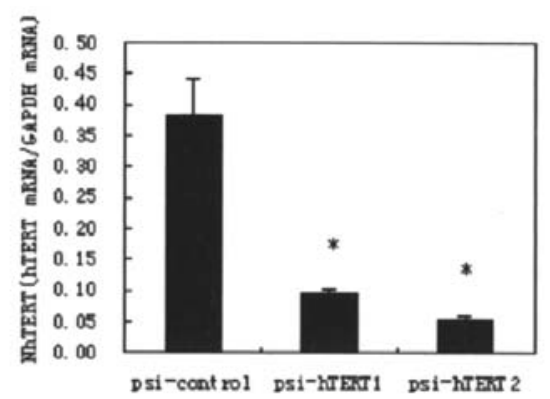

B

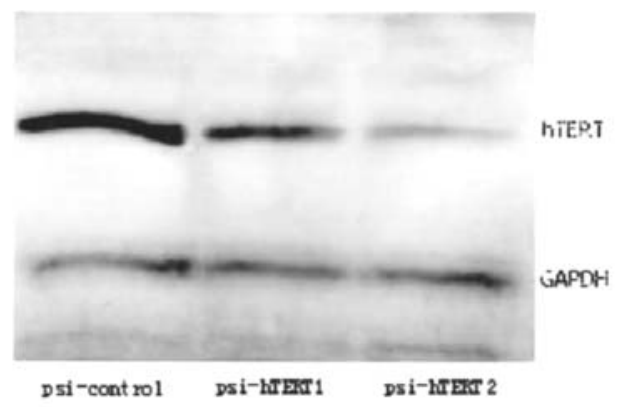

C

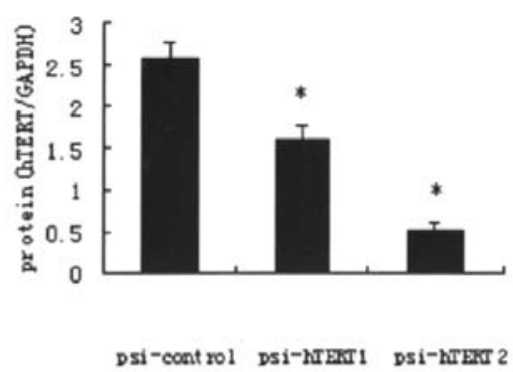

Figure 2. Effect of shRNA-targeted hTERT on hTERT gene expression. (A) Effect of shRNA-targeted hTERT on the level of hTERT mRNA expression. This was represented as NhTERT, which is the ratio of hTERT and GAPDH mRNA copies in each group. The different bands represent the mean of the results of the different groups. There was a significant difference between psi-hTERT1 or psi-hTERT2 and the psi-control; n=3 and $* \mathrm{P}<0.05$ vs. psi-control. (B) Western blotting results demonstrated the presence of hTERT and GAPDH protein among the different groups. To standardize the protein quantity, GAPDH protein was also detected by Western blotting. The upper band is the hTERT protein, while the lower band is the GAPDH protein. (C) Effect of shRNA-targeted hTERT on the level of hTERT protein expression. The bands on the film were scanned by Typhoon 9400 phosphoimager and digitized using ImageQuant software. The ratio of the hTERT versus the GAPDH expression was used as a measurement of the hTERT level. The different bands represent the mean of the results of the different groups. There was a significant difference between psi-hTERT1 or psi-hTERT2 and the psi-control; $\mathrm{n}=3$ and ${ }^{*} \mathrm{P}<0.05$ vs. psi-control. 


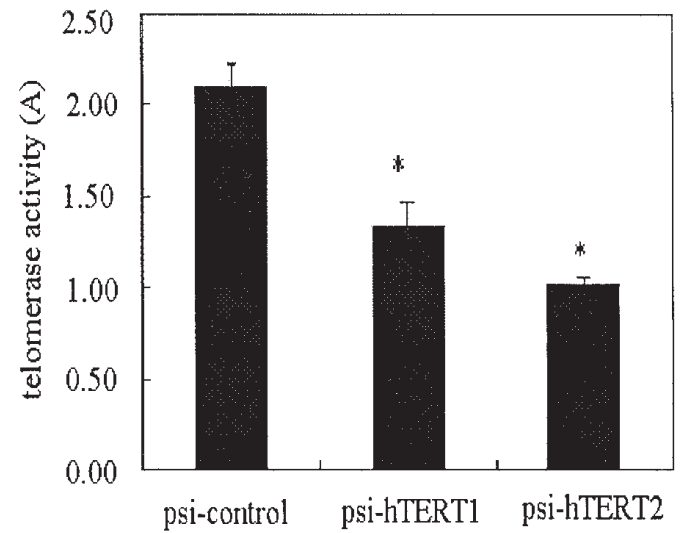

Figure 3. Effect of the shRNA-targeted hTERT on telomerase activity. Each band represents the telomerase activity of different groups. When compared with the psi-control, the telomerase activity of psi-hTERT1 and 2 was inhibited and there were significant differences between the psi-control group and the psi-hTERT1 or psi-hTERT2 group; $\mathrm{n}=3$ and ${ }^{*} \mathrm{P}<0.05$.

undergoes apoptosis (12-14). In principle, all these results indicate that telomerase inhibitors may be very useful for cancer treatment. The important advantage of using a telomerase inhibitor is that it will have far fewer side effects than many of the other cancer chemotherapy agents. Therefore, constructing a suitable telomerase inhibitor is a promising strategy for cancer tumor therapy.

Among the three components of telomerase, hTERT is the rate-limiting component of the telomerase holoenzyme $(15,16)$. The introduction of $h T E R T$ into normal human cells and the up-regulation of $h T E R T$ expression has proven that it can activate telomerase and cause cellular immortalization (17). In contrast, blocking or down-regulating the hTERT expression would lead to the shortening of the telomeres, induce chromosome instability, inhibit cell growth, accelerate cell apoptosis and alter the malignant phenotype of the cancer

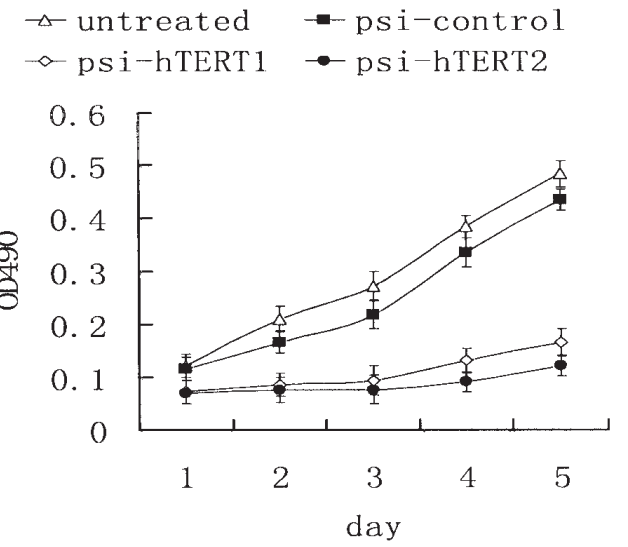

Figure 4. Growth curves of the cells of the three different groups. Cell growth was tested with MTT. Each point represented the mean of the results from three experiments. When compared with the psi-control group, cell growth markedly decreased after treatment with psi-hTERT1 or psi-hTERT2. A group of untreated cells were also shown to estimate the effect of the psicontrol group cells. There were no differences between the untreated and psi-control group.

cell (18-20). Thus, hTERT is possibly the best target among the three components for cancer therapy by inhibiting telomerase activity.

Therefore, in this study, two different 21-bp siRNAs targeted at specific hTERT fragments with different base contents were designed and separately ligated into empty shRNA expression vectors to construct shRNA-targeted hTERT-expressing vectors, which were then transfected into SGC-7901 cells to induce RNAi. Our results showed that psihTERT1 and 2 down-regulated the mRNA expression level and protein level of hTERT and correspondingly inhibited telomerase activity, increased cells apoptosis and decreased cell viability, though not to the same extent. These results agreed with the report of Akiyama et al (21). Our results also indicated that the blocking efficiency of RNAi differed across
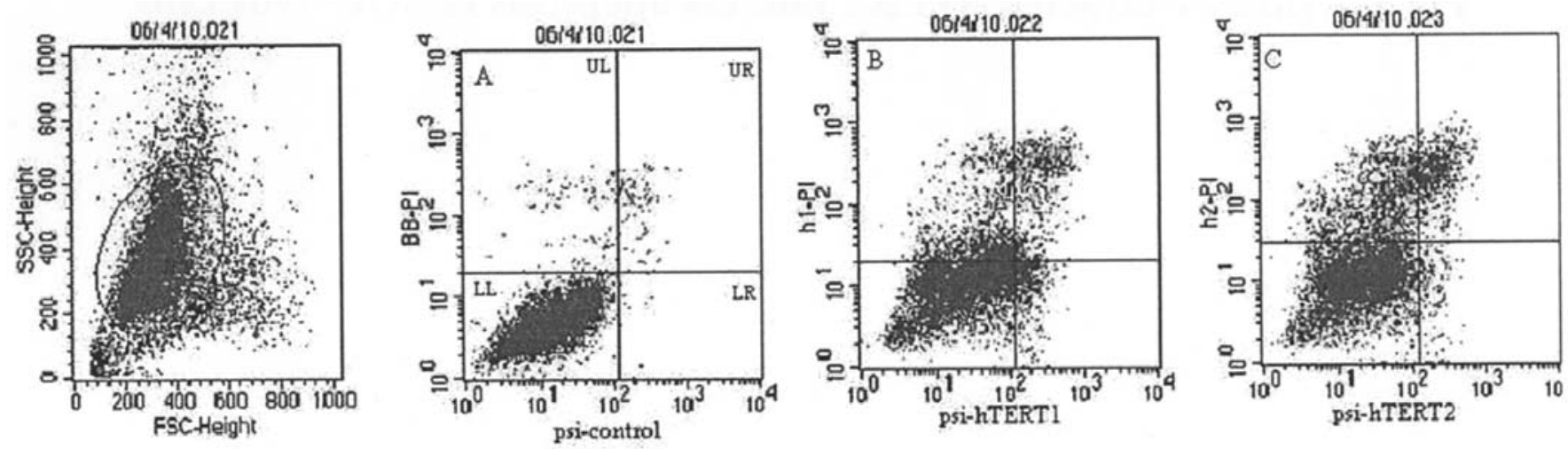

Figure 5. shRNA-targeted hTERT induces apoptosis of SGC-7901 cells. Annexin-V/PI double staining detected apoptosis in SGC-7901 cells. The four parts (LL, LR, UL and UR) represent different cell groups with different staining. The cells not stained with PI or annexin V or both (LL) were considered to be viable cells. The cells stained only with annexin V (LR) were considered to be early apoptotic. The cells stained with both PI and annexin V (UR) were considered to be late apoptotic. The cells stained only with PI (UL) were considered to be dead. Cell apoptosis was analyzed immediately with CellQuest (Version $3.1 \mathrm{f}$ ) acquired from FACSCalibur. (A) In the psi-control group, most of the cells were not stained (LL), indicating the cells were viable. The rate of cell apoptosis was $1.32 \%(\mathrm{UR}+\mathrm{LR})$ and the rate of cell viability, $97.36 \%$ (LL). (B) In the psi-hTERT1 group, the number of cells stained with annexin-V, or PI and annexin-V increased, indicating that cell apoptosis increased. The rate of cell apoptosis was $14.37 \%$ (UR + LR) and the rate of cell viability was $65.80 \%$ (LL) (C). In the psi-hTERT2 group, the number of cells stained with annexin-V, or PI and annexin-V increased. The rate of cell apoptosis was 9.88\% (UR + LR) and the rate of cell viability was $74.71 \%$ (LL). 
targeted regions, as previously proposed by Elbashir et al (22). Our results, which show that psi-hTERT2 was more effective than psi-hTERT1, suggest that further studies need to be performed to define the exact site-targeted siRNA in order to achieve a better inhibition of hTERT expression and telomerase activity.

RNAi hTERT mediated by shRNA is a prospective approach for telomerase-based cancer therapy. RNAi technology has enormous potential in silencing disease-causing genes (23) and has been applied in many disease therapies $(1-4,24)$. Studies have shown that siRNA-targeting different genes inhibited cell growth in some tumors $(25-27,29)$. However, the first obstacle in cancer gene therapy is that the silencing response induced by chemically synthesized 21-25 nt siRNAs is transient, lasting just 3-5 days in culture. This makes it impossible to analyze the long-term effects of gene silencing. Therefore, many strategies have been explored to maintain a stable effect in gene silencing (30-32). One is the use of a vector-based shRNA, whereby cells are transfected with stem-loop constructs that encode shRNAs. It is believed that there would be a stable gene-silencing effect initiated by continuously expressed shRNA. In addition, the vectors provide selectable markers, ensuring the stable expression of shRNA and silencing of the target.

A time lag exists between the telomerase inhibition and inhibition of cell proliferation, which depends on the telomeres of cancer cells (16). This requires that telomerase inhibition be maintained for a long time in cancer therapy in order to achieve better results. Some studies have reported on the short-term inhibition of telomerase activity by interfering with different sub-units of telomerase and these studies have obtained satisfactory results (33-36). However, limited detailed data exist on the long-term effect of telomerase inhibition (37). Therefore, in our studies, a DNA vectorbased RNAi system was adopted to analyze the long-term effect of hTERT gene inhibition in SGC-7901 cells. We discovered that our system effectively inhibited the expression of $h T E R T$ and telomerase activity. After the shRNA-targeted hTERT vector was transfected into SGC-7901 cells for three months, the expression levels of the hTERT mRNA and protein were significantly decreased, and the telomerase activity was also strongly inhibited in the cells. Subsequently, the growth rate of the shRNA-targeted hTERT expression vector-transfected cells decreased and they underwent apoptosis. These results suggest that shRNA-targeted hTERT has a long-term RNAi effect and this approach is more useful than the use of synthetic siRNAs for cancer gene therapy by telomerase inhibition. Despite the many difficulties, the prospect of clinical application exists.

\section{Acknowledgements}

This work was supported in part by the Nature Science Foundation of Zhenjiang City of the Jiangsu Province of China, SH2002021 and the Nature Science Foundation of the Education Ministry of Jiangsu Province of China, 04KJD320047. We thank Professors Wenrong Xu, Yongchang Chen and $\mathrm{Hu} \mathrm{Xu}$ and the lecturer Runqiu Jiang for their kind assistance in performing these experiments.

\section{References}

1. Wilda M, Fuchs U and Borkhardt A: Killing of leukemic cells with a BCR/ABL fusion gene by RNA interference (RNAi). Oncogene 21: 5716-5724, 2002.

2. Jacque JM, Triques K and Stevenson M: Modulation of HIV-1 replication by RNA interference. Nature 418: 435-438, 2002.

3. Shlomai A and Shaul Y: Inhibition of hepatitis B virus expression replication by RNA interference. Hepatology 37: 764-770, 2003.

4. Kapadia SB, Brideau-Andersen A and Chisari FV: Interference of hepatitis C virus RNA replication by short interfering RNAs. Proc Natl Acad Sci USA 100: 2014-2018, 2003.

5. Kim NW, Piatyszek MA, Prowse KR, Harley CB, West MD, Ho PL, et al: Specific association of human telomerase activity with immortal cells and cancer. Science 266: 2011-2015, 1994.

6. ABI Prism 7700 Sequence Detection System User Bulletin \#2 (2001). Relative quantification of gene expression. ttp://docs. appliedbisoystems.com/pebiodocs/04303859.pdf.

7. Tong QS, Zeng FQ, Zheng LD, Zhao J and Lu G: Apoptosis inducing effects of arsenic trioxide on human bladder cancer cell line BIU-87. Chin Med J 114: 402-406, 2001.

8. Blackburn EH: Structure and function of telomeres. Nature 350: 569-573, 1991.

9. de Lange T: Protection of mammalian telomeres. Oncogene 21: 532-540, 2002.

10. Harrington L and Robinson MO: Telomere dysfunction: Multiple paths to the same end. Oncogene 21: 592-597, 2002.

11. Morin GB: The human telomere terminal transferase enzyme is a ribonucleoprotein that synthesizes TTAGGG repeats. Cell 59: 521-529, 1989.

12. Zhang X, Mar V, Zhou W, Harrington L and Robinson MO: Telomere shortening and apoptosis in telomerase-inhibited human tumor cell. Genes Dev 13: 2388-2399, 1999.

13. de Souza Nascimento P, Alves G and Fiedler W: Telomerase inhibition by an siRNA directed against hTERT leads to telomere attrition in HT29 cells. Oncol Rep 16: 423-428, 2006.

14. Herbert BS, Pitts AE, Baker SI, Hamilton SE, Wright WE, Shay JW, et al: Inhibition of human telomerase in immortal cells lead to progressive telomere shortening and cell death. Proc Natl Acad Sci USA 96: 14276-14281, 1999.

15. Paoloni M, Gunderman JR, Estelle LN, Padilla M and Argyle DJ: Targeting the RNA component of telomerase through RNA interference and the development of vector delivery systems. Vet Comp Oncol 3: 33-34, 2005

16. Feng J, Funk WD, Wang SS, Weinrich SL, Avilion AA, Chiu CP, et al: The RNA component of human telomerase. Science 269: 1236-1241, 1995.

17. Hahn WC: Role of telomeres and telomerase in the pathogenesis of human cancer. J Clin Oncol 21: 2034-2043, 2003.

18. Masutomi K, Yu EK, Khurts S, Ben-Porath I, Currier JL, Metz GB, et al: Telomerase maintains telomere structure in normal human cells. Cell 114: 241-253, 2003.

19. Li S, Rosenberg JE, Donjacour AA, Botchkina IL, Hom YK, Cunha GR, et al: Rapid inhibition of cancer cell growth induced by lentiviral delivery and expression of mutant-template telomerase RNA and anti-telomerase short-interfering RNA. Cancer Res 64: 4833-4840, 2004.

20. Kraemer K, Fuessel S, Schmidt U, Kotzsch M, Schwenzer B, Wirth MP, et al: Antisense-mediated hTERT inhibition specifically reduces the growth of human bladder cancer cells. Clin Cancer Res 10: 3794-3800, 2003.

21. Akiyama M, Yamada O, Kanda N, Akita S, Kawano T, Onho T, et al: Telomerase overexpression in K562 leukemia cells protects against apoptosis by serum deprivation and doublestranded DNA break inducing agents, but not against DNA synthesis inhibitors. Cancer Lett 178: 187-197, 2002.

22. Elbashir SM, Lendeckel W and Tuschl T: RNA interference is mediated by -21 and -22 nucleotide RNAs. Genes Dev 15: 188-200, 2001.

23. Wall NR, Shi Y and Small RNA: Can RNA interference be exploited for therapy? Lancet 362: 1401-1403, 2003.

24. Pulukuri SM, Gondi CS, Lakka SS, Jutla A, Estes N, Gujrati M, et al: RNA interference-directed knockdown of urokinase plasminogen activator and urokinase plasminogen activator receptor inhibits prostate cancer cell invasion, survival and tumorigenicity in vivo. J Biol Chem 280: 36529-36540, 2005.

25. Zamor PD: RNA interference: Listening to the sound of silence. Nat Struct Bio 8: 746-750, 2001. 
26 Lakka SS, Gondi CS, Dinh DH, Olivero WC, Gujrati M, Rao VH, et al: Specific interference of urokinase-type plasminogen activator receptor and matrix metalloproteinase-9 gene expression induced by double-stranded RNA results in decreased invasion, tumor growth and angiogenesis in gliomas. J Biol Chem 280: 21882-21892, 2005.

27. Huang SL, Wu Y, Yu H, Zhang P, Zhang XQ, Ying L, et al: Inhibition of $\mathrm{Bcl}-2$ expression by a novel tumor-specific RNA interference system increases chemosensitivity to 5-fluorouracil in Hela cells. Acta Pharmacol Sin 27: 242-248, 2006.

28. Downward J: Use of RNA interference libraries to investigate oncogenic signaling in mammalian cells. Oncogene 23: 8376-8383, 2004.

29. Zhang MS, Zhou YF, Xie CH, Zhou F, Chen Y, Han G, et al: STAT6 specific shRNA inhibits proliferation and induces apoptosis in colon cancer HT-29 cells. Cancer Lett 243: 38-46, 2006.

30. Sui G, Soohoo C, el Affar B, Gay F, Shi Y, Forrester WC, et al: A DNA vector-based RNAi technology to suppress gene expression in mammalian cells. Proc Natl Acad Sci USA 99: 5515-5520, 2002.

31. Hu XQ, Su FX, Qin L, Jia W, Gong C, Yu F, et al: Stable RNA interference of ErbB-2 gene synergistic with epirubicin suppresses breast cancer growth in vitro and in vivo. Biochem Biophys Res Commun 346: 778-785, 2006.
32. Brummelkamp TR, Bernards R and Agami R: System for stable expression of short interfering RNAs in mammalian cells. Science 296: 550-553, 2002.

33. Xia Y, Lin RX, Zheng SJ, Yang Y, Bo XC, Zhu DY and Wang SQ: Effective siRNA targets screening for human telomerase reverse transcriptase. World J Gastroenterol 11: 2497-2501, 2005.

34. Zhang PH, Zou L and Tu ZHG: RNAi-hTERT inhibition hepatocellular carcinoma cell proliferation via decreasing telomerase activity. J Surg Res 131: 143-149, 2006.

35. Massard C, Zermati Y, Pauleau AL, Larochette N, Métivier D, Sabatier L, Kroemer G, et al: hTERT: A novel endogenous inhibitor of the mitochondrial cell death pathway. Oncogene 25: 4505-4514, 2006

36. Gandellini P, Folini M, Bandiera R, De Cesare M, Binda M, Veronese S and Daidone MG: Down-regulation of human telomerase reverse transcriptase through specific activation of RNAi pathway quickly results in cancer cell growth impairment. Biochem Pharmacol 73: 1703-1714, 2007.

37. Kurvinen K, Syrjänen S and Johansson B: Long-term suppression of telomerase expression in HeLa cell clones, transfected with an expression vector carrying siRNA targeting hTERT mRNA. Int J Oncol 29: 279-288, 2006. 